\title{
CLIDAR MOUNTAIN BOUNDARY LAYER CASE STUDIES
}

\author{
Nimmi C. P. Sharma ${ }^{1 *}$, John E. Barnes ${ }^{2}$ \\ ${ }^{I}$ Department of Physics and Engineering Physics, Central Connecticut State University, New Britain, CT \\ 06050 USA, *Email: sharmanim@ccsu.edu \\ ${ }^{2}$ National Oceanic and Atmospheric Administration, Mauna Loa Observatory, Hilo, HI USA
}

\begin{abstract}
A CCD Camera Lidar system called the CLidar system images a vertically pointing laser from the side with a spatially separated CCD camera and wide angle optics. The system has been used to investigate case studies of aerosols in mountain boundary layers in in the times following sunset. The aerosols detected by the system demonstrate the wide variation of near ground aerosol structure and capabilities of the CLidar system.
\end{abstract}

\section{INTRODUCTION}

The CCD Camera Lidar (CLidar) system is a bistatic laser radar system designed for nearground aerosol studies [1][2][3]. It has applications in aerosol profiling and boundary layer studies [4]. With the CLidar system, a laser (pulsed or continuous) is transmitted vertically into the atmosphere. The detector consists of a CCD camera equipped with a $10 \mathrm{~nm}$ laser line interference filter and wide angle optics. The detector is located far (typically $100 \mathrm{~m}$ or more) from the laser transmitter and images the entire laser beam from ground to zenith at once from the side. The signal is integrated on the CCD for several minutes. The configuration allows for submeter altitude resolution near the ground. Data are collected all the way to ground level, with no need for overlap corrections. Altitude from which the side-scatter is returned is determined by geometry from the laser and detector locations, eliminating the need for sophisticated timing electronics. The signal is influenced by laser polarization angle. Since the system is bistatic, the scattering angle varies for each altitude. Thus retrieval of aerosol extinction from the signal requires assumptions or measurements of the aerosol scattering phase function. The instrument operates only at night.

Mountain boundary layer measurements are of interest for many sites. Airflows upslope and downslope vary with time. Mauna Loa Observatory (MLO), located on the flank of
Mauna Loa volcano on the big Island of Hawaii at an altitude of $3396 \mathrm{~m}$ above sea level, is a designated Atmospheric Baseline Observatory of the National Oceanic and Atmospheric Administration's (NOAA) Global Monitoring Division (GMD), where long term atmospheric parameters are measured to determine global climate parameters. Air at this location is usually clean tropospheric air, however daytime upslope flows occur, followed by evening downslope flows. To study the atmospheric structure in the near ground region at this location, the CLidar instrument was used to map vertical aerosol distributions in the near ground region just after sunset. CLidar data were combined with data from Aerosol Robotic Network (AERONET) sunphotometers [5].

\section{METHODOLOGY}

CLidar measurements were conducted at MLO during the post-sunset period of the upslope to downslope flow transition to gain insights on variations of aerosol distributions present at these times near ground level. A linearly polarized Spectra Physics Nd:YAG model GCR-6 with 300$600 \mathrm{~mJ} /$ pulse operating at a wavelength of 532 $\mathrm{nm}$, a pulse width of $8 \mathrm{~ns}$ and a pulse repetition rate of $30 \mathrm{~Hz}$ was transmitted vertically into the atmosphere. A $10 \mathrm{~nm}$ (FWHH) laser line filter between the camera and the lens was employed to reject background light. The detector used for these experiments was a Santa Barbara Instruments Group ST-237A camera. The beam was imaged with a Kinoptic lens with a 120 degree field of view and a focal length of 1.98 $\mathrm{mm}$. The detector was located $139 \mathrm{~m}$ from the transmitter at a polarization angle of 89.4 degrees. The returned scatter signal was integrated on the camera in exposures typically of 5 minutes 32 seconds duration. CLidar experimental runs usually lasted one to two hours. Data for the case studies presented here span the months from February through August. 
Data images were analyzed using a beam finding routine which fits a function consisting of a Gaussian plus a constant to the data in the direction normal to the beam at each altitude. The constant is taken as representative of the background at each altitude. Data are normalized to match molecular scattering in a high-altitude fitting range, just as in typical backscatter lidar analysis. An aerosol phase function is then selected. The phase function shows the amount of light scattered into each angular direction. An AERONET sunphotometer [5] is available at Mauna Loa. This sunphotometer performs almucantar scans from which the overall column average aerosol scattering phase function may be derived. In the cases presented here, the AERONET-derived phase function for the daytime hours preceding the CLidar measurements was used. In the event that AERONET data are not available, phase functions from the CALIPSO phase function classes [6] could also employed. CLidar data were corrected for two way transmission, and the aerosol to molecular side scatter ratios were calculated. Lastly the aerosol extinction as a function of altitude and time were derived. This was done by using the aerosol phase function to convert from the measured side scatter to total scatter and an assumed single scattering albedo to finally derive extinction.

\section{RESULTS}

Three sample cases are presented here to demonstrate the breadth of near ground aerosol conditions encountered in this mountain boundary layer regime. Figures 1, 2 and 3 show the time averaged CLidar-derived aerosol extinction as a function of altitude for each of the three nights. Averaging times were typically 1.5 to two hours. Figures 4, 5 and 6 show the CLidar-derived aerosol extinction contour plots for the three case studies. Here the horizontal axis is the time in UTC, the vertical axis is altitude above ground level (where the instrument ground level at MLO is $3.402 \mathrm{~km}$ above sea level at the bottom of the plot), and the color map represents the derived aerosol extinction in $\mathrm{Mm}^{-1}$.

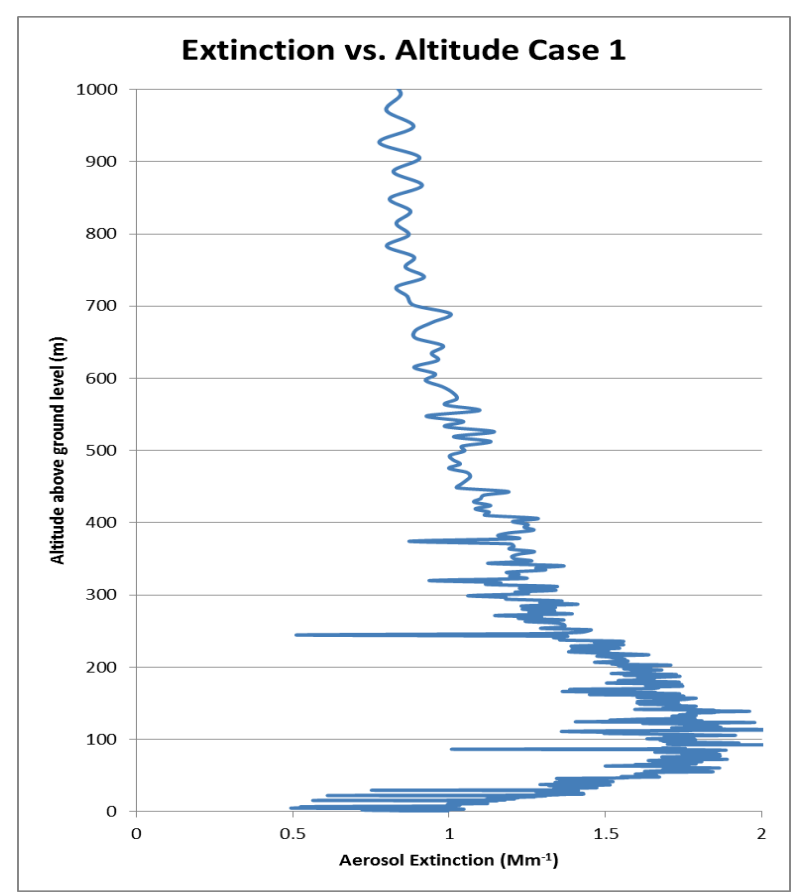

Figure 1: MLO 2/13/07 Aerosol Extinction vs. Altitude

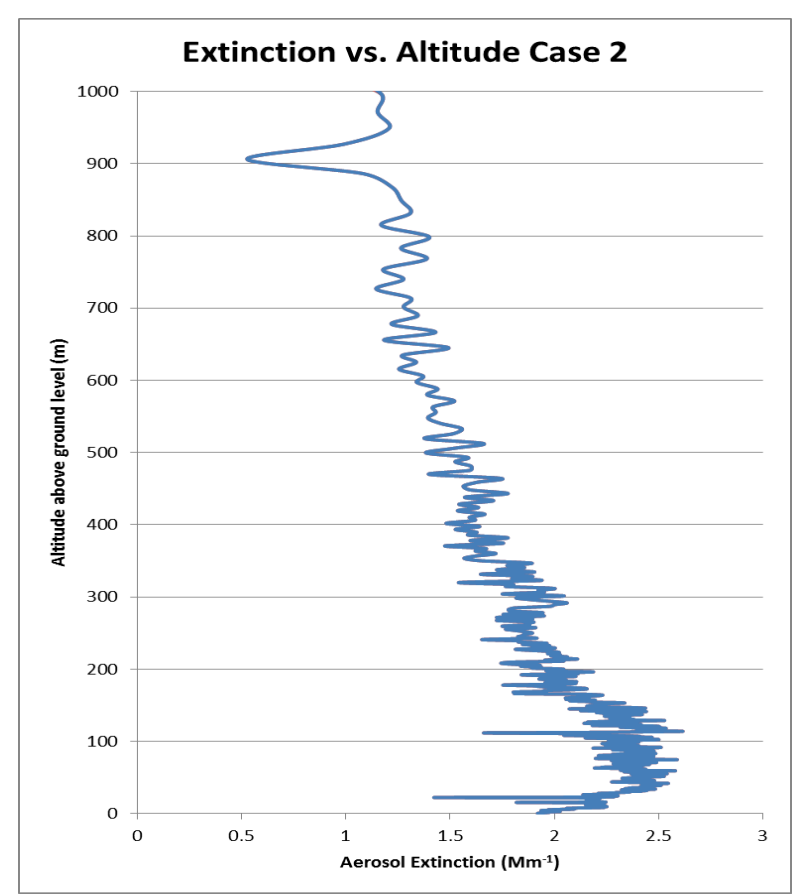

Figure 2: MLO 4/12/07 Aerosol Extinction vs. Altitude 


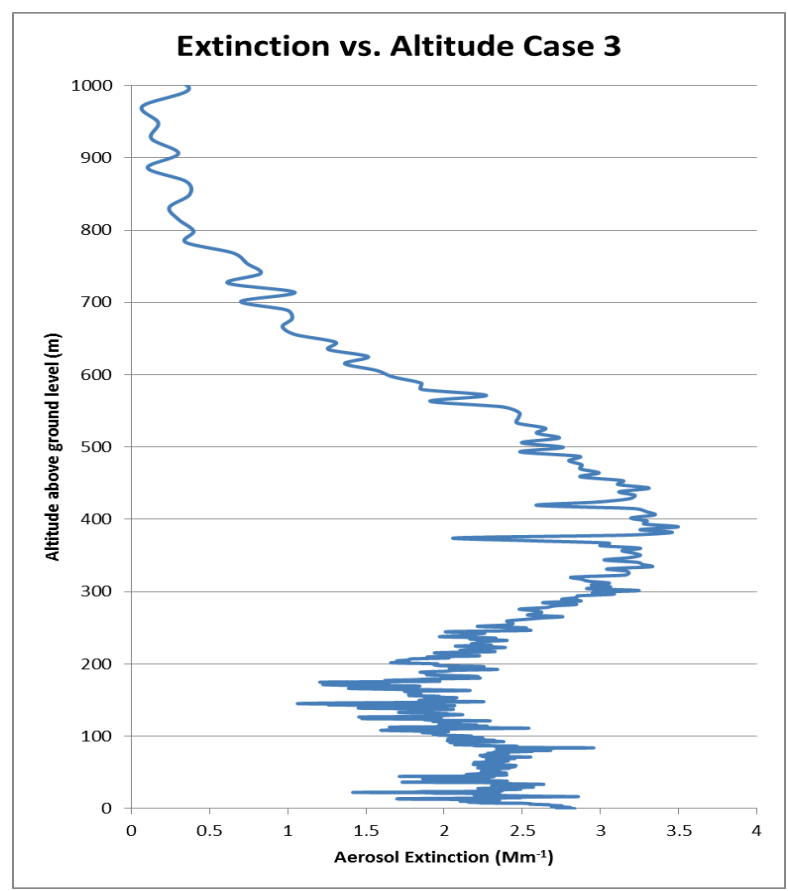

Figure 3: MLO 8/27/07 Aerosol Extinction vs. Altitude

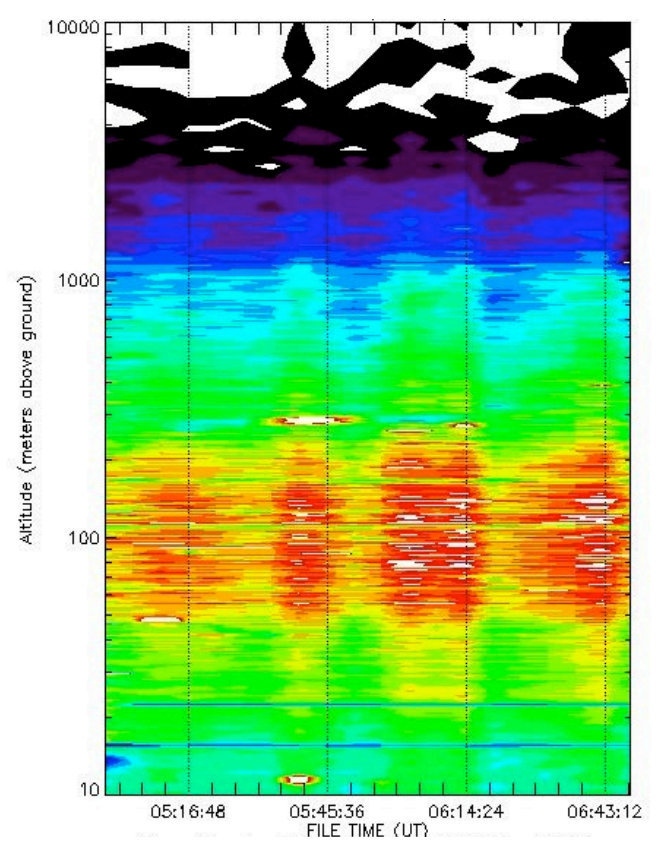

\begin{tabular}{l|l}
2.00 \\
1.87 \\
1.73 \\
1.60 \\
\hline 1.47 \\
\hline 1.33 \\
\hline 1.20 \\
\hline 1.07 \\
\hline 0.93 \\
\hline 0.60 \\
\hline 0.67 \\
\hline 0.53 \\
\hline 0.40 \\
\hline 0.27 \\
\hline 0.13 \\
\hline
\end{tabular}

Figure 4: MLO Case 1 Aerosol Extinction in $\mathrm{Mm}^{-1}$
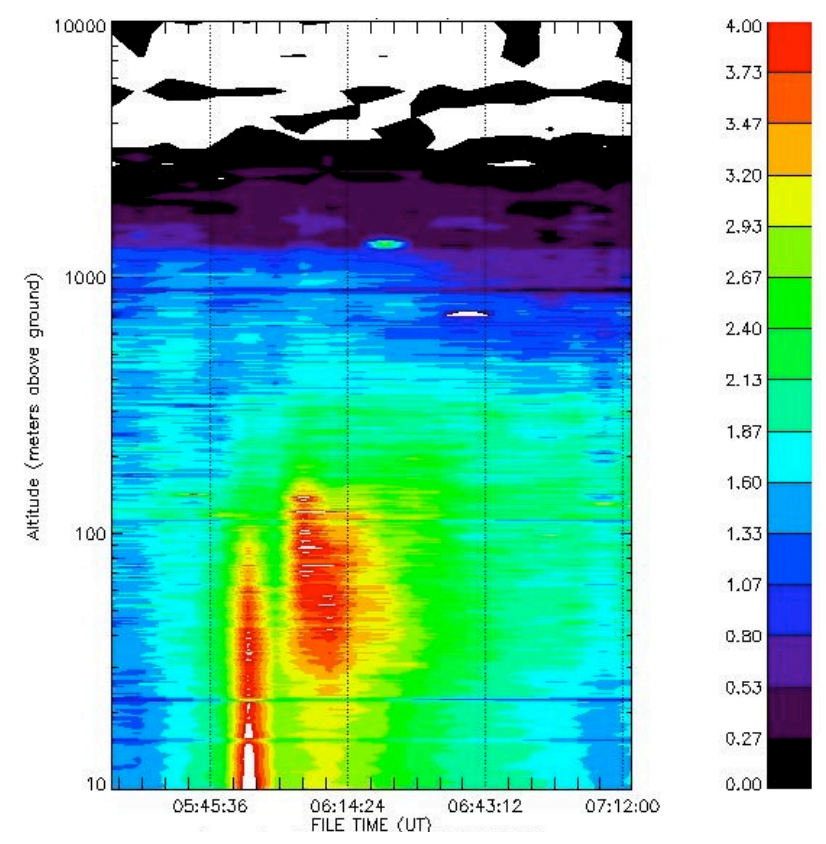

Figure 5: MLO Case 2 Aerosol Extinction in $\mathrm{Mm}^{-1}$
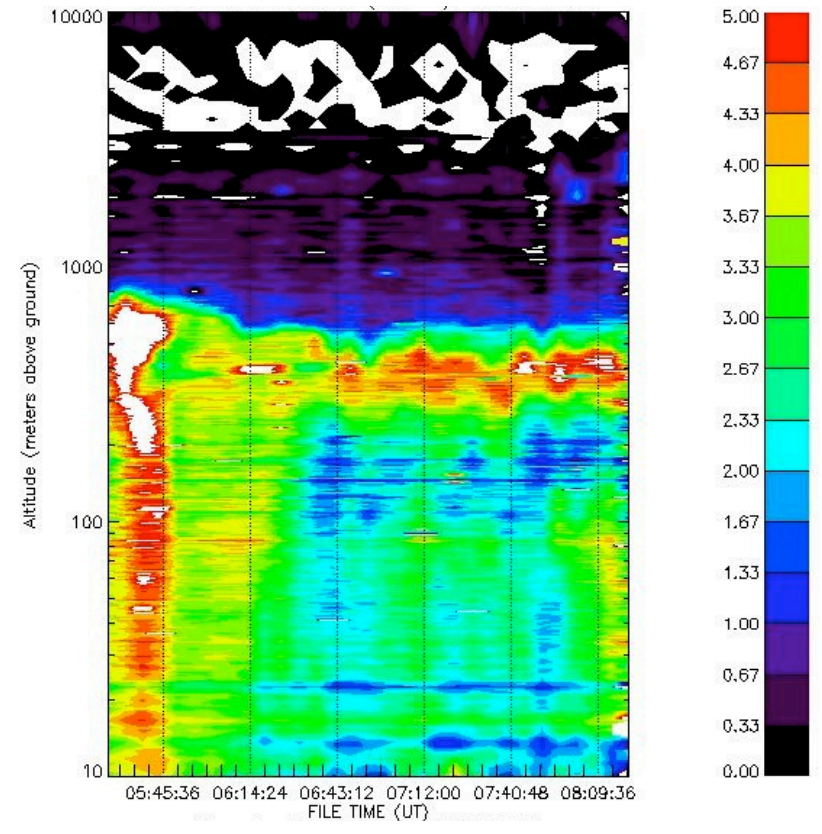

Figure 6: MLO Case 3 Aerosol Extinction in $\mathrm{Mm}^{-1}$

\section{DISCUSSION AND CONCLUSION}

All three cases presented here show low to moderate level aerosol present at Mauna Loa during the evening flow transition time. Cases of no detectable aerosol and much higher aerosol were also observed. Case 1 demonstrates persistent low-level aerosol in the near ground region. Aerosol scattering is highest at altitudes 
between 50 and 150 meters above ground level. The aerosol declines gradually at higher altitudes. Although not visible on the plot in Fig.1, the aerosol persists to an altitude of almost $3500 \mathrm{~m}$ above ground level over the experiment time frame. Case 2 shows a low passing plume of aerosol that enters the measurement region and then begins to dissipate by the end of the experimental time. The aerosol extinction at ground level changed by a factor of 3 to 4 over the 1-2 hour time frame. The aerosol varied strongly over very small distance scales as well, in some instances changing by a factor of 2 over less than $50 \mathrm{~m}$ altitude. Case 3 demonstrates an interesting example of near ground aerosol that would appear to clear out if measured only by surface instruments, while a lofted layer persists at approximately $400 \mathrm{~m}$, out of range of direct sampling instrumentation. The variation in these cases highlights the need for instrumentation capable of characterizing aerosol altitude profiles all the way to the ground. The CLidar instrument maps aerosols with high altitude resolution in this near ground region. Often the information obtained is not accessible from any other instruments. It is expected that the CLidar, combined with additional instrumentation, will be a useful tool for helping to characterize boundary layer dynamics on mountain and other sites.

\section{ACKNOWLEDGEMENT}

This material is based upon work supported by the National Science Foundation under Grant 0320800. Any opinions, findings, and conclusions or recommendations expressed in this material are the opinions of the author(s) and do not necessarily reflect the views of the National Science Foundation. The work was also supported by NOAA. The authors also acknowledge AERONET for the use of the AERONET phase function data.

\section{REFERENCES}

[1] Barnes, John E., S. Bronner, Robert Beck, and N. C. Parikh, 2003: Boundary Layer Scattering Measurements with a Charge-Coupled Device Camera Lidar, Applied Optics, 42, no. 15, 26472652.
[2] Barnes, John E., N.C. Parikh Sharma and Trevor Kaplan, Atmospheric aerosol profiling with a bistatic imaging lidar system, 2007: Applied Optics, 46, no. 15, 2922-2929.

[3] Barnes, John E. and Nimmi C. P. Sharma, 2012: An inexpensive active remote sensing instrument for assessing aerosol distributions, Journal of Air and Waste Management Association, 62, no. 2, 198-203.

[4] Sharma, N. C. Parikh, John E. Barnes, Trevor B. Kaplan and Antony D. Clarke, 2011: Coastal Aerosol Profiling With A Camera Lidar and Nephelometer, Journal of Atmospheric and Oceanic Technology (JTECH), 28, No. 3. 418425.

[5] http://aeronet.gsfc.nasa.gov/

[6] Omar, Ali H. et al., 2009: The CALIPSO automated aerosol classification and lidar ratio selection algorithm, Journal of Atmospheric and Oceanic Technology, 26, 1994-2014. 